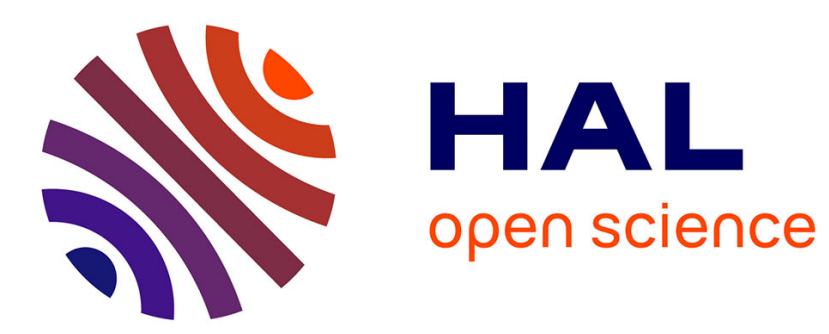

\title{
The extension to the transverse momentum of the statistical parton distributions
}

Claude Bourrely, Jacques Soffer, Franco Buccella

\section{To cite this version:}

Claude Bourrely, Jacques Soffer, Franco Buccella. The extension to the transverse momentum of the statistical parton distributions. Modern Physics Letters A, 2006, 21, pp.143-150. hal-00007731v2

\section{HAL Id: hal-00007731 \\ https://hal.science/hal-00007731v2}

Submitted on 14 Nov 2005

HAL is a multi-disciplinary open access archive for the deposit and dissemination of scientific research documents, whether they are published or not. The documents may come from teaching and research institutions in France or abroad, or from public or private research centers.
L'archive ouverte pluridisciplinaire HAL, est destinée au dépôt et à la diffusion de documents scientifiques de niveau recherche, publiés ou non, émanant des établissements d'enseignement et de recherche français ou étrangers, des laboratoires publics ou privés. 


\title{
The extension to the transverse momentum of the statistical parton distributions
}

\author{
Claude Bourrely and Jacques Soffer \\ Centre de Physique Théorique, UMR $6207^{1}$, \\ CNRS-Luminy, Case 907 \\ F-13288 Marseille Cedex 9 - France \\ Franco Buccella \\ Dipartimento di Scienze Fisiche, Università di Napoli, \\ Via Cintia, I-80126, Napoli and INFN, Sezione di Napoli, Italy
}

\begin{abstract}
By extending the statistical distributions to the transverse degree of freedhom, we account for a multiplicative factor in the Fermi-Dirac functions of the light quarks, we were led to introduce in a previous work to comply with experiment. We can also get light antiquark distributions, similar to those we proposed earlier.
\end{abstract}

keywords: polarized partons; transverse momentum; statistical models PACS numbers: 13.88+e, 13.60.Hb, 12.40.Ee

CPT-2005/P.036

UNIV. NAPLES DSF-15/2005

\footnotetext{
${ }^{1}$ UMR 6207 is Unité Mixte de Recherche du CNRS and of Universités Aix-Marseille I and Aix-Marseille II and of Université du Sud Toulon-Var, laboratoire affilié à la FRUMAN.
} 
Resulting from a research which begun many years ago [1], we proposed for the parton distributions [2], some expressions inspired by quantum statistics, with a relationship between the light $q$ and $\bar{q}$ distributions following from the chiral properties of QCD [3]. We determined the few free parameters involved, from a selected set of precise deep inelastic scattering data [2]. Our approach has a strong predictive power, because, once the parameters, occuring in the better known unpolarized $u(x)$ and $d(x)$ distributions, are fixed, the polarized distributions, $\Delta u(x)$ and $\Delta d(x)$, as well as the antiquark distributions $\bar{u}(x), \bar{d}(x), \Delta \bar{u}(x)$ and $\Delta \bar{d}(x)$, are also fixed.

First, we made a prediction for a positive $A_{1}^{n}\left(x, Q^{2}\right)$ at high $x$ [四], which was successfully verified by recent data at Jefferson Lab [5]. Next, we predicted for the high $x$ behavior of the ratio $\bar{d}(x) / \bar{u}(x)$ a monotonously increasing function of $x$. An earlier result from E866 at FNAL [6], seemed to contradict our prediction, but the comparison with the most recent results on Drell-Yan processes by the NUSEA [7], shows that this is not the case [8]. Finally, the agreement with the data on $e^{ \pm} p$ neutral and charge current reactions [9] [10], is particularly successful. In Ref. [8] we stressed the experimental evidence in several structure functions for a typical property of the statistical distributions: the change of slope for $x>X_{0 u}^{+}$, where $X_{0 u}^{+}$denotes the largest "thermodynamical potential", whose definition and value are given below. We now recall some of the basic features of the statistical approach. The fermion distributions are given by the sum of two terms [2], a quasi FermiDirac function and a helicity independent diffractive contribution equal for all light quarks:

$$
\begin{aligned}
x q^{h}\left(x, Q_{0}^{2}\right) & =\frac{A X_{0 q}^{h} x^{b}}{\exp \left[\left(x-X_{0 q}^{h}\right) / \bar{x}\right]+1}+\frac{\tilde{A} x^{\tilde{b}}}{\exp (x / \bar{x})+1}, \\
x \bar{q}^{h}\left(x, Q_{0}^{2}\right) & =\frac{\bar{A}\left(X_{0 q}^{-h}\right)^{-1} x^{2 b}}{\exp \left[\left(x+X_{0 q}^{-h}\right) / \bar{x}\right]+1}+\frac{\tilde{A} x^{\tilde{b}}}{\exp (x / \bar{x})+1},
\end{aligned}
$$

at the input energy scale $Q_{0}^{2}=4 \mathrm{GeV}^{2}$.

The parameter $\bar{x}$ plays the role of a universal temperature and $X_{0 q}^{ \pm}$are the two thermodynamical potentials of the quark $q$, with helicity $h= \pm$. For the gluons we consider the black-body inspired expression

$$
x G\left(x, Q_{0}^{2}\right)=\frac{A_{G} x^{b_{G}}}{\exp (x / \bar{x})-1},
$$


a quasi Bose-Einstein function with $b_{G}=\tilde{b}+1$, since we believe that the diffractive contribution in Eqs. $(1,2)$ is strongly related to the gluons.

We also assume that, at the input energy scale, the polarized gluon distribution vanishes, so

$$
x \Delta G\left(x, Q_{0}^{2}\right)=0 .
$$

For strange quarks we assumed that $s=\bar{s}$, but we did not introduce any more parameters and we simply related them to $\bar{u}+\bar{d}$ to comply with experimental data 11. Similarly we took $\Delta s=\Delta \bar{s}$ and we related them to $(\Delta \bar{d}-\Delta \bar{u})$, to agree with the second Bjorken sum rule.

The eight free parameters in Eqs. $(1,2)\left(A, \bar{A}\right.$ and $A_{G}$ are fixed by the normalization conditions $u-\bar{u}=2, d-\bar{d}=1$ and by the proton momentum sum rule) were determined at the input scale from the comparison with a selected set of very precise unpolarized and polarized DIS data from NMC, BCDMS, E665, ZEUS, H1 for $F_{2}^{p, d}\left(x, Q^{2}\right)$, from CCFR for $x F_{3}^{\nu N}\left(x, Q^{2}\right)$ and from SMC, E154 and E155 for $g_{1}^{p, d, n}\left(x, Q^{2}\right)$.

We obtained

$$
\begin{gathered}
\bar{x}=0.09907, b=0.40962, \tilde{b}=-0.25347, \\
X_{0 u}^{+}=0.46128, X_{0 u}^{-}=0.29766, X_{0 d}^{-}=0.30174, X_{0 d}^{+}=0.22775, \\
\tilde{A}=0.08318, A=1.74938, \bar{A}=1.90801, A_{G}=14.27535 .
\end{gathered}
$$

In Eqs. $(1,2)$ the factors $X_{0 q}^{h}$ and $\left(X_{0 q}^{-h}\right)^{-1}$ introduced in the numerators of the $q$ 's and $\bar{q}$ 's distributions, imply a modification of the quantum statistical form, we were led to introduce to agree with experimental data. As we will see now, the justification of these factors can be understood by studying the role of the transverse degree of freedhom for the partons.

Let us denote $p_{i}\left(x, p_{T}^{2}\right)^{2}$ a parton (quark or gluon) distribution with transverse momentum $p_{T}$, which is assumed to be much smaller than its longitudinal momentum $x p_{z}$, where $p_{z}$ is the momentum of the proton with mass $M$. We first consider the momentum sum rule, which reads now

$$
\sum_{i} \int_{0}^{1} d x \int_{0}^{\left(p_{T}^{2}\right)_{\max }} x p_{i}\left(x, p_{T}^{2}\right) d p_{T}^{2}=1,
$$

\footnotetext{
${ }^{2}$ Here $i$ stands for the quark flavour and for the spin component of the partons; $p_{i}\left(x, p_{T}^{2}\right)$ has now the dimension of $\mathrm{GeV}^{-2}$.
} 
where the upper limit for the $p_{T}^{2}$ integral, $\left(p_{T}^{2}\right)_{\max }$, will be determined below. Next, we consider, the condition for the energy sum rule, analogous to Eq. (8), where we assume that the proton mass is much smaller than its momentum, an approximation which holds in the deep inelastic regime. Consequently, the proton energy is $p_{z}+M^{2} / 2 p_{z}$, similarly the energy of a massless parton is $x p_{z}+p_{T}^{2} / 2 x p_{z}$, so this energy sum rule reads

$$
\begin{aligned}
& p_{z} \sum_{i} \int_{0}^{1} d x \int_{0}^{\left(p_{T}^{2}\right)_{\max }} x p_{i}\left(x, p_{T}^{2}\right) d p_{T}^{2}+ \\
& \sum_{i} \int_{0}^{1} d x \int_{0}^{\left(p_{T}^{2}\right)_{\max }} p_{i}\left(x, p_{T}^{2}\right) \frac{p_{T}^{2}}{2 x p_{z}} d p_{T}^{2}=p_{z}+\frac{M^{2}}{2 p_{z}} .
\end{aligned}
$$

By using Eq. (8) in Eq. (9), it is easy to derive the constraint

$$
\sum_{i} \int_{0}^{1} d x \int_{0}^{\left(p_{T}^{2}\right)_{\max }} p_{i}\left(x, p_{T}^{2}\right) \frac{p_{T}^{2}}{x} d p_{T}^{2}=M^{2}
$$

By comparing Eq. (10) and Eq. (8), where it is clear that $x$ is bounded by 1 , we see that $p_{T}^{2} / x$ is bounded by $M^{2}$, so $\left(p_{T}^{2}\right)_{\max }=x M^{2}$.

The above sum rule will be used to determine the $p_{T}$ dependence, as we will explain now. We recall the general method of statistical thermodynamics [12], to find the most probable occupation numbers $n_{i}$, for the energy levels $\epsilon_{i}$, when the total energy of $N$ distinguishable particles is $E$. Since we have

$$
N=\sum_{i} n_{i} \text { and } E=\sum_{i} n_{i} \epsilon_{i}
$$

one should look for the maximum of

$$
\ln \left[N ! / \prod_{i} n_{i} !\right]+\alpha\left(N-\sum_{i} n_{i}\right)+\beta\left(E-\sum_{i} n_{i} \epsilon_{i}\right)
$$

with the Lagrange multipliers method and we find

$$
n_{i}=\exp \left(-\alpha-\beta \epsilon_{i}\right)
$$

The Lagrange multipliers $\alpha$ and $\beta$ are fixed by the two above constraints Eq. (11). Now by putting in correspondence $n_{i}$ with $p_{i}\left(x, p_{T}^{2}\right), N$ with 1 and 
$\alpha$ with $1 / \bar{x}$, for the sum rule (8), and $E$ with $M^{2}, \epsilon_{i}$ with $p_{T}^{2} / x$ and $\beta$ with $1 / \mu^{2}$, for the sum rule (10), one finds, in correspondence with Eq. (13)

$$
p_{i}\left(x, p_{T}^{2}\right)=\exp \left(\frac{-x}{\bar{x}}+\frac{-p_{T}^{2}}{x \mu^{2}}\right) .
$$

$\mu$ is a parameter which has the dimension of a mass and it will be determined later. We recall that Eq. (14) describes well the $p_{T}$ behavior of the particles produced in very high energy hadron-hadron scattering [13]. For fermions, the Boltzmann exponential form Eq. (14) is expected to be modified into the product of two Fermi-Dirac expressions.

For the sake of clarity, we will first ignore the $p_{T}$ dependence of the diffractive contribution of quarks, antiquarks (second terms in Eqs. $(1,2)$ ) and of the gluons, which dominate the very low $x$ region. It certainly corresponds to very small $p_{T}$ values, since $\left(p_{T}^{2}\right)_{\max }=x M^{2}$ as seen above. So let us consider now the non-diffractive terms and instead of the helicity, as in Eqs. (1,2), the spin component of the partons along the momentum of the proton, which coincides with helicity only when $p_{T}=0$. The quantum statistics distributions for quarks and antiquarks read in this case

$$
\begin{aligned}
x q^{S_{z}}\left(x, p_{T}^{2}\right) & =\frac{F(x)}{\left[\exp \left[\left(x-X_{0 q}^{S_{z}}\right) / \bar{x}\right]+1\right]} \frac{1}{\left[\exp \left[\left(p_{T}^{2} / x \mu^{2}-Y_{0 q}^{S_{z}}\right) / \bar{x}\right]+1\right]}, \\
x \bar{q}^{S_{z}}\left(x, p_{T}^{2}\right) & =\frac{\bar{F}(x)}{\left[\exp \left[\left(x+X_{0 q}^{-S_{z}}\right) / \bar{x}\right]+1\right]} \frac{1}{\left[\exp \left[\left(p_{T}^{2} / x \mu^{2}+Y_{0 q}^{-S_{z}}\right) / \bar{x}\right]+1\right]},
\end{aligned}
$$

where $F(x)$ and $\bar{F}(x)$ do not depend on spin and flavour, $X$ and $Y$ are the thermodynamical potentials related to the sum rules (8) and (10), respectively ${ }^{3}$. At high $p_{T}$, one has a Gaussian behavior, with a width proportional to $\sqrt{x}$. It is important to note that this is at variance with the usual fatorization assumption of the dependences in $x$ and $p_{T}$ [14, 15]. We observe that the upper limit of the integral in $x$, means that one parton is taking all the proton momentum. Similarly $\left(p_{T}^{2}\right)_{\max }=x M^{2}$ for the $p_{T}^{2}$ integral corresponds to the situation where one parton is taking all the transverse energy. Therefore to simplify the formulas, we shall take as upper limit infinity, since the

\footnotetext{
${ }^{3}$ Here for convenience, we have divided by $\bar{x}$ the argument of the exponential in the Fermi-Dirac expression of the $p_{T}$ dependence, in agreement with the expression for the $x$ dependence.
} 
contribution of the tail is negligible. In fact when one now integrates over $p_{T}^{2}$, the right-hand side of Eq. (15), one finds

$$
\int_{0}^{\infty} \frac{d p_{T}^{2}}{\left.\exp \left[\left(p_{T}^{2} / x \mu^{2}-Y_{0 q}^{S_{z}}\right) / \bar{x}\right)\right]+1}=-x \mu^{2} \bar{x} \operatorname{Li}_{1}\left(-\exp \left[Y_{0 q}^{S_{z}} / \bar{x}\right]\right) .
$$

Here $\mathrm{Li}_{1}$ denotes the polylogarithm function of order 1 , which is known to arise from the integral of Fermi-Dirac distributions and is such that

$$
-\mathrm{Li}_{1}\left(-\mathrm{e}^{y}\right)=\int_{0}^{\infty} \frac{d \omega}{\mathrm{e}^{(\omega-y)}+1}=\ln \left(1+\mathrm{e}^{y}\right) .
$$

It is reasonable to assume the proportionality relationship

$$
Y_{0 q}^{S_{z}}=k X_{0 q}^{S_{z}}
$$

It implies that the partons with a larger contribution to their first moments from the non-diffractive part, not only have a broader $x$ dependence, but also have a broader $p_{T}^{2}$ dependence, at every $x$. We recall that $\mathrm{Li}_{1}$ is an increasing function of its argument and we see from Eq. (18) that for large values of $y$, it becomes approximately proportional to $y$ and one has the high degeneracy of the Fermi gas. Therefore this fully justifies, the phenomenological assumption of the proportionality to $X_{0 q}^{h}$ made in Ref. [2], as seen in Eq. (1). By taking in Eq. (15) ${ }^{4}$

$$
F(x)=-\frac{A x^{b-1} X_{0 u}^{+}}{\operatorname{Li}_{1}\left(-\exp \left[Y_{0 u}^{+} / \bar{x}\right]\right) \mu^{2} \bar{x}},
$$

we recover the first term in Eq. (1) for the $u^{+}$quark, which is the dominating parton at large $x$. Eq. (20) implies for the other quarks, that instead of $X_{0 q}^{h}$, one should use the factor $X_{0 u}^{+} \operatorname{Li}_{1}\left(-\exp \left[Y_{0 q}^{h} / \bar{x}\right]\right) / \operatorname{Li}_{1}\left(-\exp \left[Y_{0 u}^{+} / \bar{x}\right]\right)$.

As long as the non-diffractive term for the antiquarks, by integrating on $p_{T}^{2}$, one finds the factor $\operatorname{Li}_{1}\left(-\exp \left[-Y_{0 q}^{h} / \bar{x}\right]\right)$. At large negative $y, \mathrm{Li}_{1}$ becomes proportional to $\exp (-y)$, which corresponds to the Boltzman limit. Like for the quarks, we will fixe the normalization using the largest distribution, namely $\bar{d}^{-}$, so similarly to Eq. (20) we will take in Eq. (16)

$$
\bar{F}(x)=-\frac{\bar{A} x^{2 b-1}\left(X_{0 d}^{+}\right)^{-1}}{\operatorname{Li}_{1}\left(-\exp \left[-Y_{0 d}^{+} / \bar{x}\right]\right) \mu^{2} \bar{x}} .
$$

\footnotetext{
${ }^{4}$ We identify $X_{0 q}^{S_{z}}$ with $X_{0 q}^{h}$ given in Eq. (6) and similarly for the potentials $Y_{0 q}^{S_{z}}$.
} 
We recover the first term in Eq. (2) for $\bar{d}^{-}$and for the other antiquarks instead of $\left(X_{0 q}^{h}\right)^{-1}$, one should use $\left(X_{0 d}^{+}\right)^{-1} \operatorname{Li}_{1}\left(-\exp \left[-Y_{0 q}^{h} / \bar{x}\right]\right) / \operatorname{Li}_{1}\left(-\exp \left[-Y_{0 d}^{+} / \bar{x}\right]\right)$. Now, in order to determine $k$, we require no change for the quark distributions, once integrated over $p_{T}^{2}$. This can be achieved by choosing

$$
k=1.42 \text {. }
$$

This value indicates that the potentials $Y_{0 q}^{h}$ are of the same order as $X_{0 q}^{h}$ and moreover it implies lower values for the non-diffractive contributions of $\bar{u}^{+}$, $\bar{d}^{+}$and $\bar{u}^{-}$. Since the antiquark distributions are dominated by the diffractive contribution, these small modifications are still consistent with the data.

At this stage we can try to use the energy sum rule Eq. (10) to determine $\mu$, the only unknown parameter, so far. We find to a very good approximation, the remarkable value,

$$
\mu=1 \mathrm{GeV} .
$$

Moreover the energy sum rule is found to be saturated by a quark contribution of $98 \%$, whereas the antiquark contribution is only $2 \%$. This value of $\mu$ was found by neglecting the gluon and the diffractive contributions and it is clear that if they turn out to be important, $\mu$ will be smaller.

For completeness we now turn to the universal diffractive contribution to quarks and antiquarks in Eqs. $(1,2)$, namely $x q^{D}\left(x, Q_{0}^{2}\right)=\tilde{A} x^{\tilde{b}} /[\exp (x / \bar{x})+1]$. Since $\tilde{b}<0$ (see Eq. (5)), the introduction of the $p_{T}$ dependence cannot be done similarly to the non diffractive contributions, because in the energy sum rule Eq. (10), it generates a singular behavior when $x \rightarrow 0$. Therefore we propose to modify our prescription by taking at the input energy scale

$$
x q^{D}\left(x, p_{T}^{2}\right)=\frac{\tilde{A} x^{\tilde{b}-2}}{\ln (2) \mu^{2} \bar{x}} \frac{1}{[\exp (x / \bar{x})+1]} \frac{1}{\left[\exp \left(p_{T}^{2} / \bar{x} x^{2} \mu^{2}\right)+1\right]},
$$

whose $p_{T}$ fall off is stronger, because $x \mu^{2}$ is now replaced by $x^{2} \mu^{2}$. Note that this is properly normalized to recover $x q^{D}\left(x, Q_{0}^{2}\right)$ after integration over $p_{T}^{2}$. We have checked that $x q^{D}\left(x, p_{T}^{2}\right)$ gives a negligible contribution to Eq. (10), as expected, and we are led to a similar conclusion for the gluon, which has the same small $x$ behavior. However since the gluon is parametrized by a quasi Bose-Einstein function, one has to introduce a non-zero potential $Y_{G}$, to avoid the singular behavior of $\operatorname{Li}_{1}\left(\exp \left[-Y_{G} / \bar{x}\right]\right)$, when $Y_{G}=0$ (see Eq. (18)). The value of $Y_{G}$ is not constrained, but by taking a very small $Y_{G}$, it does not affect the energy sum rule. Of course, one may ask why the 
gluon contributes so much to the momentum sum rule, Eq. (8), and so little to the energy sum rule, Eq. (10). Using a better regularization procedure, we might find a larger contribution and consequently a smaller $\mu$ value. For this reason, we give the $p_{T}$ dependence as a function of the dimensionless variable $p_{T}^{2} / \mu^{2}$.

For illustration, we display in Fig. $1, x \mu^{2} u\left(x, p_{T}^{2} / \mu^{2}, Q_{0}^{2}\right)$, the predicted dimensionless $u$-quark parton distribution at input energy scale, versus $p_{T}^{2} / \mu^{2}$ for different $x$ values. Note that the curves are limited to the value $\left(p_{T}^{2}\right)_{\max }=$ $x M^{2}$ and the Gaussian behavior in the high $p_{T}$ region follows a rather flat behavior for $10^{-2}<p_{T}^{2} / \mu^{2}<10^{-1}$, or so. This feature is a natural consequence of the statistical approach and it coincides, for a mean $x$ value, with the simplifying assumption made in recent papers (see e.g. [13]) without real justification. For $p_{T}^{2} / \mu^{2}<10^{-2}$, the very low $x$ region is strongly enhanced by the diffractive contribution, due to the factor $x^{\tilde{b}-2}$ (see Eq. (24)). However, since the regularization procedure proposed above is not unique, it should not be considered as a definite prediction.

The theoretical fundation of the statistical parton distributions proposed earlier in Ref. [2] is strengthened by the present extension to the transverse degrees of freedhom. Indeed the energy sum rule Eq. (10) cuts naturally high $p_{T}^{2}$ values and this is very appealing, because an increasing phase-space for $p_{T}^{2}$ linear in $Q^{2}$ would support the absence of quantum statistical effects for diluted fermions and bosons. Our predicted light partons distributions $p_{i}\left(x, p_{T}^{2}\right)$, both unpolarized and polarized given by Eqs. $(15,16)$, remain to be checked against appropriate experimental data, sensitive to some effects arising from the transverse momentum of partons inside the proton. Here it is important to emphasize that the $p_{T}$ dependence is only valid in a limited kinematic region, say $0.1<x<0.9$, which is not dominated by the diffractive contribution. Clearly in this large $x$ region, we predict the mean value of $p_{T}^{2}$, namely $<p_{T}^{2}>$, to increase with $x$, a behaviour which is at variance with what one expects in the very small $x$ region from the BFKL evolution, assuming the emission of a hudge number of gluons [16]. However, so far there is no experimental evidence for the BFKL evolution.

Finally, we note that our procedure restricts to rotational invariance, the transverse momentum dependence of the parton distributions. The next step should be to introduce some azimuthal dependence, which is needed to study the important topic of single-spin asymmetries [17. 


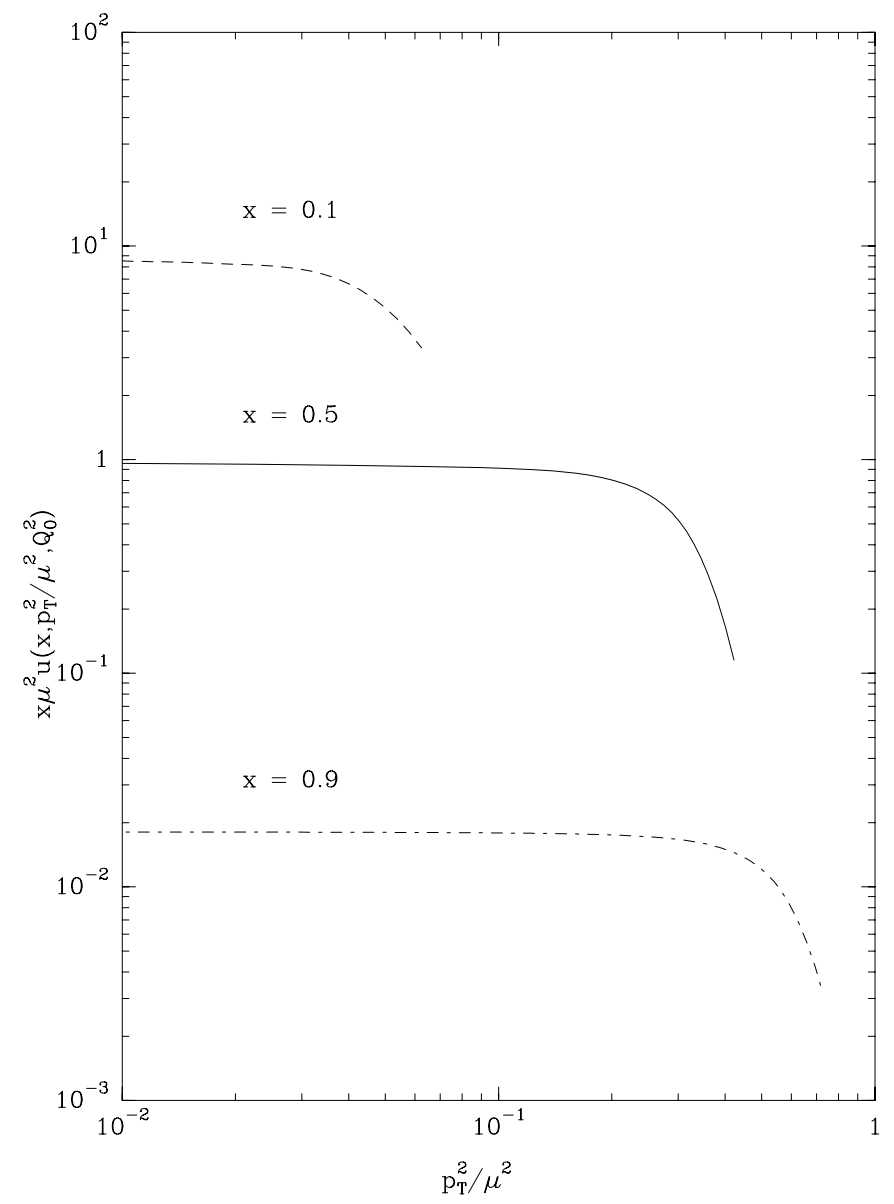

Figure 1: The predicted $x \mu^{2} u\left(x, p_{T}^{2} / \mu^{2}, Q_{0}^{2}\right)$ statistical distribution, versus $p_{T}^{2} / \mu^{2}$ for different values of $x$. 


\section{References}

[1] C. Bourrely, F. Buccella, G. Miele, G. Migliore, J. Soffer and V. Tibullo, Z. Phys. C 62, 431 (1994)

[2] C. Bourrely, F. Buccella and J. Soffer, Eur. Phys. J. C 23, 487 (2002)

[3] R.S. Bhalerao, Phys. Rev. C 63, 025208 (2001)

[4] C. Bourrely, F. Buccella and J. Soffer, Mod. Phys. Lett. 18, 771 (2003)

[5] Jefferson Lab Hall A Collaboration, X. Zheng et al., Phys. Rev. C 70, 065207 (2004)

[6] FNAL Nusea Collaboration, E. A. Hawker et al., Phys. Rev. Lett. 80, 3715 (1998); J. C. Peng et al., Phys. Rev. D 58, 092004 (1998); R. S. Towell et al., Phys. Rev. D 64, 052002 (2001)

[7] FNAL E866/NuSea Collaboration, J.C. Webb et al., submitted to Phys. Rev. Lett. [hep-ex/0302019]

[8] C. Bourrely, F. Buccella and J. Soffer, Eur. Phys. J. C 41, 327 (2005)

[9] H1 Collaboration, C. Adloff et al., Eur. Phys. J. C 30, 1 (2003), ibid 19, 269 (2001)

[10] ZEUS Collaboration, S. Chekanov et al., Eur. Phys. J. C 21, 443 (2001); Eur. Phys. J. C 28, 175 (2003); Phys. Rev. D 70, 052001 (2004); Eur. Phys. J. C 32, 1 (2003); Phys. Lett. B539, 197 (2002)

[11] CCFR Collaboration, P. Z. Quintas et al., Phys. Rev. Lett. 71, 1307 (1993); W. C. Leung et al., Phys. Lett. B 317, 655 (1993); W. G. Seligman et al., Phys. Rev. Lett. 79, 1213 (1997); J. H. Kim et al., Phys. Rev. Lett. 81, 3595 (1998); U. K. Yang et al., Phys. Rev. Lett. 86, 2741 (2001)

[12] E. Schrödinger, Statistical Thermodynamics, Cambridge University Press (1964).

[13] F. Buccella and L. Popova, Mod. Phys. Lett. A 17, 2627 (2002)

[14] M. Anselmino, U. D'Alesio and F. Murgia, Phys. Rev. D 67, 074010 (2003) 
[15] I. Schmidt, J. Soffer and J.J. Yang, Phys. Lett. B 612, 258 (2005)

[16] The Small $x$ Collaboration, J.R. Andersen et al., Eur. Phys. J. C 35, 67 (2004) and references therein

[17] D. Boer, P.J. Mulders and F. Pijlman, Nucl. Phys. B 667, 201 (2003) 